\title{
Global Attractors for a Class of Generalized Nonlinear Kirchhoff-Sine-Gordon Equation
}

\author{
Ruijin Lou, Penghui Lv, Guoguang Lin \\ Mathematical of Yunnan University, Kunming, China \\ Email: 1145068367@qq.com, 18487279097@163.com,gglin@ynu.edu.cn
}

Received 29 December 2015; accepted 14 March 2016; published 17 March 2016

Copyright (C) 2016 by authors and Scientific Research Publishing Inc.

This work is licensed under the Creative Commons Attribution International License (CC BY). http://creativecommons.org/licenses/by/4.0/

\section{(c) (i) Open Access}

\section{Abstract}

In this paper, we consider a class of generalized nonlinear Kirchhoff-Sine-Gordon equation $u_{t t}-\beta \Delta u_{t}+\alpha u_{t}-\phi\left(\|\nabla u\|^{2}\right) \Delta u+g(\sin u)=f(x)$. By a priori estimation, we first prove the existence and uniqueness of solutions to the initial boundary value conditions, and then we study the global attractors of the equation.

\section{Keywords}

Kirchhoff-Sine-Gordon Equation, The Existence and Uniqueness of Solutions, Priori Estimates, Global Attractors

\section{Introduction}

In 1883, Kirchhoff [1] proposed the following model in the study of elastic string free vibration:

$u_{t t}-\alpha \Delta u-M\left(\|\nabla u\|^{2}\right) \Delta u=f(x, u)$, where $\alpha$ is associated with the initial tension, $M$ is related to the material properties of the rope, and $u(x, t)$ indicates the vertical displacement at the $x$ point on the $t$. The equation is more accurate than the classical wave equation to describe the motion of an elastic rod.

Masamro [2] proposed the Kirchhoff equation with dissipation and damping term:

$$
\begin{cases}u_{t t}-M\left(\|\nabla u\|^{2}\right) \Delta u+\delta|u|^{p} u+\gamma u_{t}=f(x) & x \in \Omega, t>0 \\ u(x, t)=0 & x \in \partial \Omega, t \geq 0 \\ u(x, 0)=u_{0}(x), u_{t}(x, 0)=u_{1}(x) & x \in \Omega\end{cases}
$$

How to cite this paper: Lou, R.J., Lv, P.H. and Lin, G.G. (2016) Global Attractors for a Class of Generalized Nonlinear Kirchhoff-Sine-Gordon Equation. International Journal of Modern Nonlinear Theory and Application, 5, 73-81. 
where $\Omega$ is a bounded domain of $R^{n}(n \geq 1)$ with a smooth boundary $\partial \Omega$; he uses the Galerkin method to prove the existence of the solution of the equation at the initial boundary conditions.

Sine-Gordon equation is a very useful model in physics. In 1962, Josephson [3] fist applied the Sine-Gordon equation to superconductors, where the equation: $u_{t t}-u_{x x}+\sin u=0, u_{t t}$ is the two-order partial derivative of $u$ with respect to the variable $t ; u_{x x}$ is the two-order partial derivative of the $u$ about the independent variable $x$. Subsequently, Zhu [4] considered the following problem: $u_{t t}-\alpha u_{t}-u_{x x}+\lambda g(\sin u)=f(x, t)$ (where $\Omega$ is a bounded domain of $R^{3}$ ) and he proved the existence of the global solution of the equation. For more research on the global solutions and global attractors of Kirchhoff and sine-Gordon equations, we refer the reader to [5]-[11].

Based on Kirchhoff and Sine-Gordon model, we study the following initial boundary value problem:

$$
\begin{cases}u_{t t}-\beta \Delta u_{t}+\alpha u_{t}-\phi\left(\|\nabla u\|^{2}\right) \Delta u+g(\sin u)=f(x) & \\ u(x, t)=0 & x \in \partial \Omega, t \geq 0 \\ u(x, 0)=u_{0}(x), u_{t}(x, 0)=u_{1}(x) & x \in \Omega\end{cases}
$$

where $\Omega$ is a bounded domain of $R^{n}(n \geq 1)$ with a smooth boundary $\partial \Omega ; \alpha$ is the dissipation coefficient; $\beta$ is a positive constant; and $f(x)$ is the external interference. The assumptions on nonlinear terms $g(\sin u)$ and $\phi\left(\|\nabla u\|^{2}\right)$ will be specified later.

The rest of this paper is organized as follows. In Section 2, we first obtain the basic assumption. In Section 3, we obtain a priori estimate. In Section 4, we prove the existence of the global attractors.

\section{Basic Assumption}

For brevity, we define the Sobolev space as follows:

$$
\begin{gathered}
H=L^{2}(\Omega), V_{1}=H_{0}^{1}(\Omega), V_{2}=H^{2}(\Omega) \cap H_{0}^{1}(\Omega), \\
E_{0}=H_{0}^{1}(\Omega) \times L^{2}(\Omega)=V_{1} \times H, E_{1}=\left(H^{2}(\Omega) \cap H_{0}^{1}(\Omega)\right) \times H_{0}^{1}(\Omega)=V_{2} \times V_{1} .
\end{gathered}
$$

In addition, we define $(\bullet, \bullet)$ and $\|\bullet\|$ are the inner product and norm of $H$.

Nonlinear function $g(s)$ satisfying condition $(G)$ :

(1) $g(s) \in C^{2}(R)$;

(2) $|g(s)| \leq c\left(1+|s|^{p}\right)$;

(3) $\left|\frac{\mathrm{d} g(s)}{\mathrm{d} s}\right| \leq c\left(1+|s|^{p-1}\right)$, where $c>0,1 \leq p \leq \frac{2 n}{n-2}, n \geq 3$.

Function $\phi(s)$ satisfies the condition $(F)$ :

(4) $\phi(s) \in C^{1}([0,+\infty), R)$;

(5) $\frac{m_{1}+2}{2}<m_{0} \leq \phi(s) \leq m_{1}, 0 \leq \frac{\mathrm{d} \phi(s)}{\mathrm{d} s} \leq c_{0}$;

(6) $\Phi(s)=\int_{0}^{u} \phi(\tau) \mathrm{d} \tau$;

(7) $\phi(s) s \geq c_{1} \Phi(s)$, where $c_{1} \geq \frac{2(m+1)}{m_{0}}, m=\left\{\begin{array}{l}m_{0}, \frac{\mathrm{d}}{\mathrm{d} t}\|\Delta u\|^{2} \geq 0 \\ m_{1}, \frac{\mathrm{d}}{\mathrm{d} t}\|\Delta u\|^{2}<0 .\end{array}\right.$

\section{A Priori Estimates}

Lemma 3.1. Assuming the nonlinear function $g(s), \phi(s)$ satisfies the condition $(G)-(F), \quad\left(u_{0}, u_{1}\right) \in V_{1} \times H$, 
$f \in H, v=u_{t}+\varepsilon u, 0<\varepsilon \leq \min \left\{\frac{\alpha}{4}, \frac{m_{0}}{2 \beta}, \frac{2 m_{0}-m-2}{3 \beta}\right\}$, then the solution $(u, v)$ of the initial boundary value problem (1.1) satisfies $(u, v) \in V_{1} \times H$ and

$$
\|(u, v)\|_{V_{1} \times H}^{2}=\|\nabla u\|^{2}+\|v\|^{2} \leq \frac{y_{1}(0)}{k_{1}} \mathrm{e}^{-\alpha_{1} t}+\frac{c_{2}}{\alpha_{1} k_{1}}\left(1-\mathrm{e}^{-\alpha_{1} t}\right),
$$

where $y_{1}(0)=\|v(0)\|^{2}+\Phi\left(\|\nabla u(0)\|^{2}\right)-\beta \varepsilon\|\nabla u(0)\|^{2}$. Thus there exists a positive constant $c\left(R_{0}\right)$ and $t_{1}=t_{1}(\Omega)>0$, such that

$$
\|(u, v)\|_{V_{1} \times H}^{2}=\|\nabla u(t)\|^{2}+\|v(t)\|^{2} \leq c\left(R_{0}\right)\left(t>t_{1}\right) .
$$

Proof. Let $v=u_{t}+\varepsilon u$, the equation $u_{t t}-\beta \Delta u_{t}+\alpha u_{t}-\phi\left(\|\nabla u\|^{2}\right) \Delta u+g(\sin u)=f(x)$ can be transformed into

$$
v_{t}+(\alpha-\varepsilon) v+\varepsilon(\varepsilon-\alpha) u+\beta \varepsilon \Delta u-\beta \Delta v-\phi\left(\|\nabla u\|^{2}\right) \Delta u+g(\sin u)=f(x) .
$$

Taking the inner product of the equations (3.1) with $v$ in $H$, we find that

$$
\begin{aligned}
& \frac{1}{2} \frac{\mathrm{d}}{\mathrm{d} t}\|v\|^{2}+(\alpha-\varepsilon)\|v\|^{2}+\varepsilon(\varepsilon-\alpha)(u, v)-\frac{\beta \varepsilon}{2} \frac{\mathrm{d}}{\mathrm{d} t}\|\nabla u\|^{2} \\
& -\beta \varepsilon^{2}\|\nabla u\|^{2}+(-\beta \Delta v, v)+\left(-\phi\left(\|\nabla u\|^{2}\right) \Delta u, v\right)=(f-g(\sin u), v) .
\end{aligned}
$$

By using Holder inequality, Young's inequality and Poincare inequality, we deal with the terms in (3.2) one by as follows

$$
(-\beta \Delta v, v)=\beta(\nabla v, \nabla v)=\beta\|\nabla u\|^{2} \geq \lambda_{1} \beta\|u\|^{2},
$$

where $\lambda_{1}$ is the first eigenvalue of $-\Delta$ with Dirichlet boundary conditions on $\Omega$.

Since $0<\varepsilon \leq \frac{\alpha}{4}$ and (F) (6), (7), we get

$$
\begin{aligned}
\varepsilon(\varepsilon-\alpha)(u, v) & \geq \frac{\varepsilon(\varepsilon-\alpha)}{\sqrt{\lambda_{1}}}\|\nabla u\|\|v\| \geq-\frac{\varepsilon \alpha}{\sqrt{\lambda_{1}}}\left(\frac{\sqrt{\lambda_{1}}}{\alpha}\|\nabla u\|^{2}+\frac{\alpha}{\sqrt{\lambda_{1}}}\|v\|^{2}\right) \\
& \geq-\varepsilon\|\nabla u\|^{2}-\frac{\alpha^{3}}{4 \lambda_{1}}\|v\|^{2},
\end{aligned}
$$

and

$$
\begin{gathered}
\left(-\phi\left(\|\nabla u\|^{2}\right) \Delta u, v\right)=\frac{\phi\left(\|\nabla u\|^{2}\right)}{2} \frac{\mathrm{d}}{\mathrm{d} t}\|\nabla u\|^{2}+\varepsilon \phi\left(\|\nabla u\|^{2}\right)\|\nabla u\|^{2} \\
\geq \frac{1}{2} \frac{\mathrm{d}}{\mathrm{d} t} \Phi\left(\|\nabla u\|^{2}\right)+c_{1} \varepsilon \Phi\left(\|\nabla u\|^{2}\right) . \\
(f-g(\sin u), v) \leq\|v\|(\|f\|+\|g(\sin u)\|) \leq \frac{\alpha}{2}\|v\|^{2}+\frac{\left(\|f\|+2 c|\Omega|^{\frac{1}{2}}\right)^{2}}{2 \alpha},
\end{gathered}
$$

where

$$
\|g(\sin u)\| \leq\left(\int_{\Omega}\left|c\left(1+|\sin u|^{p}\right)\right|^{2} \mathrm{~d} x\right)^{\frac{1}{2}} \leq 2 c|\Omega|^{\frac{1}{2}} .
$$

Combined (3.1)-(3.6) type, it follows from that 


$$
\begin{aligned}
& \frac{\mathrm{d}}{\mathrm{d} t}\left[\|v\|^{2}+\Phi\left(\|\nabla u\|^{2}\right)-\beta \varepsilon\|\nabla u\|^{2}\right]+\left(\alpha+2 \lambda_{1} \beta-\frac{\alpha^{3}}{2 \lambda_{1}}-2 \varepsilon\right)\|v\|^{2} \\
& +2 c_{1} \varepsilon \Phi\left(\|\nabla u\|^{2}\right)+2 \varepsilon(-\beta \varepsilon-1)\|\nabla u\|^{2} \leq \frac{\left(\|f\|+2 c|\Omega|^{\frac{1}{2}}\right)^{2}}{\alpha}=c_{2} .
\end{aligned}
$$

According to condition (F) (5), this will imply $m_{0}\|\nabla u\|^{2} \leq \Phi\left(\|\nabla u\|^{2}\right) \leq m_{1}\|\nabla u\|^{2}$, then, $\Phi\left(\|\nabla u\|^{2}\right)-\beta \varepsilon\|\nabla u\|^{2}>0$, and since $c_{1} \geq \frac{2(m+1)}{m_{0}}$,

$$
\begin{aligned}
c_{1} \Phi\left(\|\nabla u\|^{2}\right)+(-\beta \varepsilon-1)\|\nabla u\|^{2} & \geq \Phi\left(\|\nabla u\|^{2}\right)-\beta \varepsilon\|\nabla u\|^{2}+\left(m_{0}-1\right)\|\nabla u\|^{2} \\
& \geq \Phi\left(\|\nabla u\|^{2}\right)-\beta \varepsilon\|\nabla u\|^{2}
\end{aligned}
$$

that is

$$
2 \varepsilon\left[c_{1} \Phi\left(\|\nabla u\|^{2}\right)+(-\beta \varepsilon-1)\|\nabla u\|^{2}\right]>\varepsilon\left[\Phi\left(\|\nabla u\|^{2}\right)-\beta \varepsilon\|\nabla u\|^{2}\right] .
$$

With (3.10), (3.8) can be written as

$$
\begin{aligned}
& \frac{\mathrm{d}}{\mathrm{d} t}\left[\|v\|^{2}+\Phi\left(\|\nabla u\|^{2}\right)-\beta \varepsilon\|\nabla u\|^{2}\right]+\left(\alpha+2 \lambda_{1} \beta-\frac{\alpha^{3}}{2 \lambda_{1}}-2 \varepsilon\right)\|v\|^{2} \\
& +\varepsilon\left[\Phi\left(\|\nabla u\|^{2}\right)-\beta \varepsilon\|\nabla u\|^{2}\right] \leq c_{2} .
\end{aligned}
$$

Set $a=\alpha+2 \lambda_{1} \beta-\frac{\alpha^{3}}{2 \lambda_{1}}-2 \varepsilon \geq 0$, and $\alpha_{1}=\min \{a, \varepsilon\}$, then (3.11) is equivalent to (3.12)

$$
\frac{\mathrm{d}}{\mathrm{d} t} y_{1}(t)+\alpha_{1} y_{1}(t) \leq c_{2}
$$

where

$$
y_{1}(t)=\|v\|^{2}+\Phi\left(\|\nabla u\|^{2}\right)-\beta \varepsilon\|\nabla u\|^{2} .
$$

By using Gronwall inequality, we obtain

$$
y_{1}(t) \leq y_{1}(0) \mathrm{e}^{-\alpha_{1} t}+\frac{c_{2}}{\alpha_{1}}\left(1-\mathrm{e}^{-\alpha_{1} t}\right) .
$$

Let $k_{1}=\min \left\{1,\left(m_{0}-\beta \varepsilon\right)\right\}$.

So, we have

$$
\|(u, v)\|_{V_{1} \times H}^{2}=\|\nabla u\|^{2}+\|v\|^{2} \leq \frac{y_{1}(0)}{k_{1}} \mathrm{e}^{-\alpha_{1} t}+\frac{c_{2}}{\alpha_{1} k_{1}}\left(1-\mathrm{e}^{-\alpha_{1} t}\right),
$$

then

$$
\varlimsup_{t \rightarrow \infty}\|(u, v)\|_{V_{1} \times H}^{2} \leq \frac{c_{2}}{\alpha_{1} k_{1}} .
$$

Hence, there exists $c\left(R_{0}\right)$ and $t_{1}=t_{1}(\Omega)>0$, such that

$$
\|(u, v)\|_{V_{1} \times H}^{2}=\|\nabla u(t)\|^{2}+\|v(t)\|^{2} \leq c\left(R_{0}\right)\left(t>t_{1}\right) .
$$


Lemma 3.2. Assuming the nonlinear function $g(s), \phi(s)$ satisfies the condition $(G)-(F)$, $\left(u_{0}, u_{1}\right) \in V_{2} \times V_{1}, f \in V_{1}, v=u_{t}+\varepsilon u, 0<\varepsilon \leq \min \left\{\frac{\alpha}{4}, \frac{m_{0}}{2 \beta}, \frac{2 m_{0}-m-2}{3 \beta}\right\}$, then the solution $(u, v)$ of satisfies the initial boundary value problem (1.1) satisfies $(u, v) \in V_{2} \times V_{1}$ and

$$
\|(u, v)\|_{V_{2} \times V_{1}}^{2}=\|\nabla v\|^{2}+\|\Delta u\|^{2} \leq \frac{y_{2}(0)}{k_{2}} \mathrm{e}^{-\alpha_{2} t}+\frac{c_{3}}{\alpha_{2} k_{2}}\left(1-\mathrm{e}^{-\alpha_{2} t}\right),
$$

where $y_{2}(0)=\|\nabla v(0)\|^{2}+(m-\beta \varepsilon)\|\Delta u(0)\|^{2}$. Thus there exists a positive constant $c\left(R_{1}\right)$ and $t_{2}=t_{2}(\Omega)>0$, such that

$$
\|(u, v)\|_{V_{2} \times V_{1}}^{2}=\|\nabla v(t)\|^{2}+\|\Delta u(t)\|^{2} \leq c\left(R_{1}\right)\left(t>t_{1}\right) .
$$

Proof. The equations (3.1) in the $H$ and $-\Delta v=-\Delta u_{t}-\varepsilon \Delta u$ have inner product, we find that

$$
\begin{aligned}
& \frac{1}{2} \frac{\mathrm{d}}{\mathrm{d} t}\|\nabla v\|^{2}+(\alpha-\varepsilon)\|\nabla v\|^{2}+\varepsilon(\varepsilon-\alpha)(u,-\Delta v)-\frac{\beta \varepsilon}{2} \frac{\mathrm{d}}{\mathrm{d} t}\|\Delta u\|^{2}-\beta \varepsilon^{2}\|\Delta u\|^{2} \\
& +(-\beta \Delta v,-\Delta v)+\left(-\phi\left(\|\nabla u\|^{2}\right) \Delta u,-\Delta v\right)=(f-g(\sin u),-\Delta v) .
\end{aligned}
$$

By using Holder inequality, Young's inequality and Poincare inequality, we get the following results

$$
\begin{gathered}
(-\beta \Delta v,-\Delta v)=\beta(\Delta v, \Delta v)=\beta\|\Delta v\|^{2} \geq \lambda_{1} \beta\|\nabla v\|^{2}, \\
\varepsilon(\varepsilon-\alpha)(u,-\Delta v) \geq \frac{\varepsilon^{2}-\varepsilon \alpha}{\sqrt{\lambda_{1}}}\|\Delta u\|\|\nabla v\| \geq-\frac{\varepsilon \alpha}{\sqrt{\lambda_{1}}}\left(\frac{\sqrt{\lambda_{1}}}{\alpha}\|\Delta u\|^{2}+\frac{\alpha}{\sqrt{\lambda_{1}}}\|\nabla v\|^{2}\right) \\
\geq-\varepsilon\|\Delta u\|^{2}-\frac{\alpha^{3}}{4 \lambda_{1}}\|\nabla v\|^{2} .
\end{gathered}
$$

According to condition (F) (5), (6), we obtain

$$
\begin{aligned}
\left(-\phi\left(\|\nabla u\|^{2}\right) \Delta u,-\Delta v\right) & =\phi\left(\|\nabla u\|^{2}\right)(\Delta u, \Delta v)=\phi\left(\|\nabla u\|^{2}\right)\left[\left(\Delta u, \Delta u_{t}\right)+(\Delta u, \varepsilon \Delta u)\right] \\
& =\frac{\phi\left(\|\nabla u\|^{2}\right)}{2} \frac{\mathrm{d}}{\mathrm{d} t}\|\Delta u\|^{2}+\varepsilon \phi\left(\|\nabla u\|^{2}\right)\|\Delta u\|^{2} \geq \frac{m}{2} \frac{\mathrm{d}}{\mathrm{d} t}\|\Delta u\|^{2}+\varepsilon m_{0}\|\Delta u\|^{2} . \\
(f-g(\sin u),-\Delta v) \leq\|\nabla v\|(\|\nabla f\|+\|\nabla g(\sin u)\|) \leq \frac{\alpha}{2}\|\nabla v\|^{2}+\frac{(\|\nabla f\|+\|\nabla g(\sin u)\|)^{2}}{2 \alpha} & \\
& \leq \frac{\alpha}{2}\|\nabla v\|^{2}+\frac{\left(\|\nabla f\|+2 c|\Omega|^{\frac{1}{2}}\right)^{2}}{2 \alpha},
\end{aligned}
$$

where

$$
\begin{aligned}
\|\nabla g(\sin u)\| & =\left\|g^{\prime}(\sin u) \cos u \nabla u\right\| \leq\left\|g^{\prime}(\sin u)\right\|\|\nabla u\| \\
& \leq R_{1}\left(\int_{\Omega}\left|c\left(1+|\sin u|^{p-1}\right)\right|^{2} \mathrm{~d} x\right)^{\frac{1}{2}} \leq 2 R_{1} c|\Omega|^{\frac{1}{2}} .
\end{aligned}
$$

By (3.18)-(3.22), (3.17) can be written

$$
\begin{aligned}
& \frac{\mathrm{d}}{\mathrm{d} t}\left[\|\nabla v\|^{2}+(m-\beta \varepsilon)\|\Delta u\|^{2}\right]+\left(\alpha+2 \lambda_{1} \beta-2 \varepsilon-\frac{\alpha^{3}}{2 \lambda_{1}}\right)\|\nabla v\|^{2} \\
& +2 \varepsilon\left(m_{0}-\beta \varepsilon-1\right)\|\Delta u\|^{2} \leq \frac{\left(\|\nabla f\|+2 c R_{1}|\Omega|^{\frac{1}{2}}\right)^{2}}{\alpha}=c_{3} .
\end{aligned}
$$


Noticing $0<\varepsilon \leq \frac{2 m_{0}-m-2}{3 \beta}$, this will imply

$$
2 \varepsilon\left(m_{0}-\beta \varepsilon-1\right)\|\Delta u\|^{2} \geq \varepsilon(m-\beta \varepsilon)\|\Delta u\|^{2} .
$$

Substituting (3.24) into (3.23), we can get the following inequality

$$
\frac{\mathrm{d}}{\mathrm{d} t}\left[\|\nabla v\|^{2}+(m-\beta \varepsilon)\|\Delta u\|^{2}\right]+\left(\alpha+2 \lambda_{1} \beta-2 \varepsilon-\frac{\alpha^{3}}{2 \lambda_{1}}\right)\|\nabla v\|^{2}+\varepsilon(m-\beta \varepsilon)\|\Delta u\|^{2} \leq c_{3} .
$$

Let $b=\alpha+2 \lambda_{1} \beta-2 \varepsilon-\frac{\alpha^{3}}{2 \lambda_{1}} \geq 0$, and $\alpha_{2}=\min \{b, \varepsilon\}$, then (3.25) type can be changed into

$$
\frac{\mathrm{d}}{\mathrm{d} t}\left[\|\nabla v\|^{2}+(m-\beta \varepsilon)\|\Delta u\|^{2}\right]+\alpha_{2}\left[\|\nabla v\|^{2}+(m-\beta \varepsilon)\|\Delta u\|^{2}\right] \leq c_{3},
$$

then

$$
\frac{\mathrm{d}}{\mathrm{d} t} y_{2}(t)+\alpha_{2} y_{2}(t) \leq c_{3}
$$

where $y_{2}(t)=\|\nabla v\|^{2}+(m-\beta \varepsilon)\|\Delta u\|^{2}$.

By using Gronwall inequality, we obtain

$$
y_{2}(t) \leq y_{2}(0) \mathrm{e}^{-\alpha_{2} t}+\frac{c_{3}}{\alpha_{2}}\left(1-\mathrm{e}^{-\alpha_{2} t}\right),
$$

taking $k_{2}=\min \{1,(m-\beta \varepsilon)\}$, we have

$$
\|(u, v)\|_{V_{2} \times V_{1}}^{2}=\|\Delta u\|^{2}+\|\nabla v\|^{2} \leq \frac{y_{2}(0)}{k_{2}} \mathrm{e}^{-\alpha_{2} t}+\frac{c_{3}}{\alpha_{2} k_{2}}\left(1-\mathrm{e}^{-\alpha_{2} t}\right),
$$

then

$$
\varlimsup_{t \rightarrow \infty}\|(u, v)\|_{V_{2} \times V_{1}}^{2} \leq \frac{c_{3}}{\alpha_{2} k_{2}} .
$$

Hence, there exists $c\left(R_{1}\right)$ and $t_{2}=t_{2}(\Omega)>0$, such that

$$
\|(u, v)\|_{V_{2} \times V_{1}}^{2}=\|\Delta u(t)\|^{2}+\|\nabla v(t)\|^{2} \leq c\left(R_{1}\right)\left(t>t_{2}\right) .
$$

Theorem 3.1. Assuming the nonlinear function $g(s), \phi(s)$ satisfies the condition $(G)-(F)$,

$\left(u_{0}, u_{1}\right) \in V_{2} \times V_{1}, f \in V_{1}, v=u_{t}+\varepsilon u, \quad 0<\varepsilon \leq \min \left\{\frac{\alpha}{4}, \frac{m_{0}}{2 \beta}, \frac{2 m_{0}-m-2}{3 \beta}\right\}$, so the initial boundary value problem (1.1) exists a unique smooth solution $(u, v) \in L^{\infty}\left([0,+\infty) ; V_{2} \times V_{1}\right)$.

Proof. By Lemma 3.1-Lemma 3.2 and Glerkin method, we can easily obtain the existence of solutions of equation $(u, v) \in L^{\infty}\left([0,+\infty) ; V_{2} \times V_{1}\right)$, the proof procedure is omitted. Next, we prove the uniqueness of solutions in detail.

Assume $u, v$ are two solutions of equation, we denote $w=u-v$, then, the two equations subtract and obtain

$$
w_{t t}-\beta \Delta w_{t}+\alpha w_{t}-\phi\left(\|\nabla u\|^{2}\right) \Delta u+\phi\left(\|\nabla v\|^{2}\right) \Delta v=-g(\sin u)+g(\sin v) .
$$

We take the inner product of the above equations (3.31) with $w_{t}$ in $H$, we have 


$$
\begin{aligned}
& \frac{1}{2} \frac{\mathrm{d}}{\mathrm{d} t}\left\|w_{t}\right\|^{2}+\left(-\beta \Delta w_{t}, w_{t}\right)+\alpha\left\|w_{t}\right\|^{2}+\left(-\phi\left(\|\nabla u\|^{2}\right) \Delta u+\phi\left(\|\nabla v\|^{2}\right) \Delta v, w_{t}\right) \\
& =\left(-g(\sin u)+g(\sin v), w_{t}\right) .
\end{aligned}
$$

We deal with the terms in (3.32) one by as follows

$$
\left(-\beta \Delta w_{t}, w_{t}\right)=\beta\left(\nabla w_{t}, \nabla w_{t}\right)=\beta\left\|\nabla w_{t}\right\|^{2} \geq \lambda_{1} \beta\left\|w_{t}\right\|^{2},
$$

and

$$
\begin{aligned}
& \left(-\phi\left(\|\nabla u\|^{2}\right) \Delta u+\phi\left(\|\nabla v\|^{2}\right) \Delta v, w_{t}\right) \\
& =\left(-\phi\left(\|\nabla u\|^{2}\right) \Delta u+\phi\left(\|\nabla u\|^{2}\right) \Delta v-\phi\left(\|\nabla u\|^{2}\right) \Delta v+\phi\left(\|\nabla v\|^{2}\right) \Delta v, w_{t}\right) \\
& =-\phi\left(\|\nabla u\|^{2}\right)\left(\Delta u-\Delta v, w_{t}\right)+\left(-\phi\left(\|\nabla u\|^{2}\right)+\phi\left(\|\nabla v\|^{2}\right)\right)\left(\Delta v, w_{t}\right) \\
& =\frac{m}{2} \frac{\mathrm{d}}{\mathrm{d} t}\|\nabla w\|^{2}+\left(-\phi\left(\|\nabla u\|^{2}\right)+\phi\left(\|\nabla v\|^{2}\right)\right)\left(\Delta v, w_{t}\right) .
\end{aligned}
$$

By (3.32)-(3.34), we can get the following inequality

$$
\begin{aligned}
& \frac{1}{2} \frac{\mathrm{d}}{\mathrm{d} t}\left\|w_{t}\right\|^{2}+\lambda_{1} \beta\left\|w_{t}\right\|^{2}+\alpha\left\|w_{t}\right\|^{2}+\frac{m}{2} \frac{\mathrm{d}}{\mathrm{d} t}\|\nabla w\|^{2} \\
& =\left(\phi\left(\|\nabla u\|^{2}\right)-\phi\left(\|\nabla v\|^{2}\right)\right)\left(\Delta v, w_{t}\right)+\left(-g(\sin u)+g(\sin v), w_{t}\right) .
\end{aligned}
$$

Further, by mid-value theorem and Young's inequality, we get

$$
\begin{aligned}
& \left(\phi\left(\|\nabla u\|^{2}\right)-\phi\left(\|\nabla v\|^{2}\right)\right)\left(\Delta v, w_{t}\right) \leq \mid \phi^{\prime}(\eta)(\|\nabla u\|+\|\nabla v\|)\|\nabla w\|\|\Delta v\|\left\|w_{t}\right\| \\
& \leq c_{0}(\|\nabla u\|+\|\nabla v\|)\|\nabla w\|\|\Delta v\|\left\|w_{t}\right\| \leq c_{4}\|\nabla w\|\left\|w_{t}\right\| \leq \frac{c_{4}}{2 \lambda_{1}}\|\nabla w\|^{2}+\frac{\lambda_{1} c_{4}}{2}\left\|w_{t}\right\|^{2} .
\end{aligned}
$$

Since $\|g(\sin v)-g(\sin u)\|^{2}=\int_{\Omega}\left(\frac{g(\sin v)-g(\sin u)}{\sin v-\sin u}\right)^{2} \cdot(\sin v-\sin u)^{2} \mathrm{~d} x=\int_{\Omega}\left(g^{\prime}(\gamma)\right)^{2}(\sin v-\sin u)^{2} \mathrm{~d} x$, might as well set $\gamma=\theta \sin v+(1-\theta) \sin u(0 \leq \theta \leq 1)$.

$$
\begin{aligned}
\|g(\sin v)-g(\sin u)\|^{2} & \leq \int_{\Omega}\left[c\left(1+|\theta \sin v+(1-\theta) \sin u|^{p-1}\right)\right]^{2}(\sin v-\sin u)^{2} \mathrm{~d} x \\
& \leq c^{2}\left(1+2^{p-1}\right)^{2} \int_{\Omega}(\sin v-\sin u)^{2} \mathrm{~d} x \leq c_{5}^{2}\|u-v\|^{2},
\end{aligned}
$$

where $c_{5}=c\left(1+2^{p-1}\right)$.

Then, we obtain

$$
\left(-g(\sin u)+g(\sin v), w_{t}\right) \leq c_{5}\|w\|\left\|w_{t}\right\| \leq \frac{c_{5}}{2 \lambda_{1}}\|\nabla w\|^{2}+\frac{c_{5}}{2}\left\|w_{t}\right\|^{2} .
$$

Substituting (3.36), (3.37) into (3.35), we can get

$$
\frac{\mathrm{d}}{\mathrm{d} t}\left(\left\|w_{t}\right\|^{2}+m\|\nabla w\|^{2}\right) \leq \frac{c_{4}+c_{5}}{\lambda_{1}}\|\nabla w\|^{2}+\left(c_{5}+\lambda_{1} c_{4}\right)\left\|w_{t}\right\|^{2} .
$$

Let $k=\max \left\{\left(c_{5}+\lambda_{1} c_{4}\right), \frac{c_{4}+c_{5}}{\lambda_{1} m}\right\}$, then (3.38) can be changed to

$$
\frac{\mathrm{d}}{\mathrm{d} t}\left(\left\|w_{t}\right\|^{2}+m\|\nabla w\|^{2}\right) \leq k\left(\left\|w_{t}\right\|^{2}+m\|\nabla w\|^{2}\right) .
$$


By using Gronwall inequality, we obtain

$$
\left\|w_{t}(t)\right\|^{2}+m\|\nabla w(t)\|^{2} \leq\left\|w_{t}(0)\right\|^{2}+m\|\nabla w(0)\|^{2} \mathrm{e}^{k t}=0 .
$$

There has

$$
\left\|w_{t}(t)\right\|^{2}+m\|\nabla w(t)\|^{2} \leq 0
$$

That show that $w_{t}(t)=0, \nabla w(t)=0$.

So as to get $w(t) \equiv 0, u=v$, the uniqueness is proved.

\section{Global Attractor}

Theorem 4.1. [12] Set $E_{1}$ be a Banach space, and $\{S(t)\}(t \geq 0)$ are the semigroup operator on $E_{1}$. $S(t): E_{1} \rightarrow E_{1}, S(t+s)=S(t) S(s)(\forall t, s \geq 0), S(0)=I$; here $I$ is a unit operator. Set $S(t)$ satisfy the follow conditions.

1) $S(t)$ is bounded, namely $\forall R>0,\|u\|_{E_{1}} \leq R$; it exists a constant $c(R)$, so that

$$
\|S(t) u\|_{E_{1}} \leq c(R)(t \in[0,+\infty)) ;
$$

2) It exists a bounded absorbing set $B_{0} \subset E_{1}$, namely, $\forall B \subset E_{1}$; it exists a constant $t_{0}$, so that

$$
S(t) B \subset B_{0}\left(t \geq t_{0}\right) \text {; }
$$

here $B_{0}$ and $B$ are bounded sets.

3) When $t>0, S(t)$ is a completely continuous operator.

Therefore, the semigroup operators $S(t)$ exist a compact global attractor $A$.

Theorem 4.2. [12] Under the assume of Theorem 3.1, equations have global attractor

$$
A=\omega\left(B_{0}\right)=\bigcap_{s \geq 0} \overline{\bigcup_{t \geq s} S(t) B_{0}},
$$
where $B_{0}=\left\{(u, v) \in V_{2} \times V_{1}:\|(u, v)\|_{V_{2} \times V_{1}}^{2}=\|u\|_{V_{2}}^{2}+\|v\|_{V_{1}}^{2} \leq c\left(R_{0}\right)+c\left(R_{1}\right)\right\} ; \quad B_{0}$ is the bounded absorbing set of
$V_{2} \times V_{1}$ and satisfies

(1) $S(t) A=A, t>0$;

(2) $\lim _{t \rightarrow \infty} \operatorname{dist}(S(t) B, A)=0$, here $B \subset V_{2} \times V_{1}$ and it is a bounded set, $\operatorname{dist}(S(t) B, A)=\operatorname{supinf}_{x \in B}\|S \in A(t) x-y\|_{V_{2} \times V_{1}}$.

Proof. Under the conditions of Theorem 3.1, it exists the solution semigroup $S(t)$, here $E_{1}=V_{2} \times V_{1}, S(t): E_{1} \rightarrow E_{1}$.

(1) From Lemma 3.1-Lemma 3.2, we can get that $\forall B \subset V_{2} \times V_{1}$ is a bounded set that includes in the ball $\left\{\|(u, v)\|_{V_{2} \times V_{1}} \leq R\right\}$,

$$
\left\|S(t)\left(u_{0}, v_{0}\right)\right\|_{V_{2} \times V_{1}}^{2}=\|u\|_{V_{2}}^{2}+\|v\|_{V_{1}}^{2} \leq\left\|u_{0}\right\|_{V_{2}}^{2}+\left\|v_{0}\right\|_{V_{1}}^{2}+c \leq R^{2}+c,\left(t \geq 0,\left(u_{0}, v_{0}\right) \in B\right) .
$$

This shows that $S(t)(t \geq 0)$ is uniformly bounded in $V_{2} \times V_{1}$.

(2) Furthermore, for any $\left(u_{0}, v_{0}\right) \in V_{2} \times V_{1}$, when $t \geq \max \left\{t_{1}, t_{2}\right\}$, we have

$$
\left\|S(t)\left(u_{0}, v_{0}\right)\right\|_{V_{2} \times V_{1}}^{2}=\|u\|_{V_{2}}^{2}+\|v\|_{V_{1}}^{2} \leq c\left(R_{1}\right)+c\left(R_{2}\right) .
$$

So we get $B_{0}$ is the bounded absorbing set.

(3) Since $V_{2} \times V_{1} \rightarrow V_{1} \times H$ is compact embedded, which means that the bounded set in $V_{2} \times V_{1}$ is the compact set in $V_{1} \times H$, so the semigroup operator $S(t)$ is completely continuous.

Hence, the semigroup operator $S(t)$ exists a compact global attractor $A$. The proving is completed. 


\section{Acknowledgements}

The authors express their sincere thanks to the anonymous reviewer for his/her careful reading of the paper, giving valuable comments and suggestions. These contributions greatly improved the paper.

\section{Funding}

This work is supported by the National Natural Sciences Foundation of People’s Republic of China under Grant 11161057.

\section{References}

[1] Kirchhof, G. (1883) Vorlesungen fiber Mechanik. Teubner, Stuttgarty.

[2] Masamro, H. and Yoshio, Y. (1991) On Some Nonlinear Wave Equations 2: Global Existence and Energy Decay of Solutions. J. Fac. Sci. Univ. Tokyo. Sect. IA, Math., 38, 239-250.

[3] Josephson, B.D. (1962) Possible New Effects in Superconductive Tunneling. Physics Letters, 1, 251-253. http://dx.doi.org/10.1016/0031-9163(62)91369-0

[4] Zhu, Z.W. and Lu, Y. (2000) The Existence and Uniqueness of Solution for Generalized Sine-Gordon Equation. Chinese Quarterly Journal of Mathematics, 15, 71-77.

[5] Li, Q.X. and Zhong, T. (2002) Existence of Global Solutions for Kirchhoff Type Equations with Dissipation and Damping Terms. Journal of Xiamen University: Natural Science Edition, 41, 419-422.

[6] Silva, M.A.J. and Ma, T.F. (2013) Long-Time Dynamics for a Class of Kirchhoff Models with Memory. Journal of Mathematical Physics, 54, Article ID: 021505.

[7] Zhang, J.W., Wang, D.X. and Wu, R.H. (2008) Global Solutions for a Class of Generalized Strongly Damped SineGordon Equation. Journal of Mathematical Physics, 57, 2021-2025.

[8] Guo, L., Yuan, Z.Q. and Lin, G.G. (2014) The Global Attractors for a Nonlinear Viscoelastic Wave Equation with Strong Damping and Linear Damping and Source Terms. International Journal of Modern Nonlinear Theory and Application, 4, 142-152. http://dx.doi.org/10.4236/ijmnta.2015.42010

[9] Teman, R. (1988) Infiniter-Dimensional Dynamical Systems in Mechanics and Physics. Springer-Verlag, New York, 15-26. http://dx.doi.org/10.1007/978-1-4684-0313-8 2

[10] Ma, Q.F., Wang, S.H. and Zhong, C.K. (2002) Necessary and Sufficient Congitions for the Existence of Global Attractors for Semigroup and Applications. Indiana University Mathematics Journal, 51, 1541-1559. http://dx.doi.org/10.1512/iumj.2002.51.2255

[11] Ma, Q.Z., Sun, C.Y. and Zhong, C.K. (2007) The Existence of Strong Global Attractors for Nonlinear Beam Equations. Journal of Mathematical Physics, 27A, 941-948.

[12] Lin, G.G. (2011) Nonlinear Evolution Equation. Yunnan University Press, 12. 